\title{
Editorial
}

\section{Europäische Euphorien}

Nach dem Zweiten Weltkrieg ging ein Bild durch die Presse und später in die Lehrbücher der Sozialkunde ein: junge europäische Föderalisten beseitigen Schlagbäume an den Grenzen, natürlich nur symbolisch. Nach dem Zweiten Weltkrieg, der vor allem ein europäischer Krieg war, sollte damit zum Ausdruck gebracht werden, daß mit den Grenzen zwischen den europäischen Kleinstaaten auch die Konflikte zwischen ihnen abzuschaffen seien. Erst der Sieg über Hitler-Deutschland hatte den alten Gedanken an eine europäische Versöhnung zur neuen Wirklichkeit gedrängt. Die Grenzen blieben - bis heute, doch ihre Höhe wurde schrittweise verringert: und mit der Schaffung des gemeinsamen Marktes Ende 1992 sollen sie definitiv fallen. Der Traum der Coudenhove-Kalergi scheint sich noch in diesem Jahrhundert zu erfüllen.

Die Europabegeisterung in den 40ern hat sich in den 80er Jahren zu einem ansteckenden Europa-Fieber gesteigert: Intellektuelle $»$ träumen « Europa und meinen damit ein sozial, weltanschaulich, kulturell und sprachlich vielgestaltiges, grenzenloses und begeisterndes Gebilde. Die Industriellen machen es - unter tatkräftiger Mithilfe der Euro-Bürokratie - und denken dabei eher an die Vereinheitlichung von Normen und Steuersätzen und an die Harmonisierung von Reglements. Von europäischen Freiheiten ist in beiden Diskursen die Rede. Die einen haben dabei »europäische Traditionen « im Sinn: die Autonomie der Kunst, die Würde des freien Individuums, die uneingelösten Versprechen der Französischen Revolution - allgemeine Ideen von Freiheit, Gleichheit und Solidarität. Die anderen phantasieren Freiheiten eher in den traditionellen Dimensionen des privaten Eigentums und den modernen Visionen eines grenzenlosen Supermarktes: Freizügigkeit für Dienstleistungen, Waren, Kapital und Arbeitskräfte. Europäisch versteht sich das auf großen Tagungen in Lissabon, Berlin oder Mailand inszenierte kollektive Räsonnement der Intellektuellen, weil (und solange) es der Rationalität der Aufklärung verpflichtet ist. Eine Vorstellung vom Fortschritt des Wissens, vor allem der technologischen Erfahrung aber besitzen auch die, denen eher die Ökonomie Europas am Herzen knapp unterhalb der Brieftasche liegt; mit ihrem Vertrauen auf die Rationalität einer zentralen Bürokratie sind sie dem Geiste der Aufklärung am Ende des zweiten Milleniums womöglich näher als diejenigen, die seit 200 Jahren die trinitarische Formel der Französischen Revolution hochhalten. Jedenfalls verfolgen sie mit ihrem Europa des freien Marktes nicht das Projekt einer »sozialen Demokratie « in kontinentalem Ausmaß, das zum Beispiel Peter Glotz herbeizureden versucht, - - sondern erst einmal die Harmonisierung von rund 5000 Normen; auf daß der Philips-Konzern, statt wie derzeit 30 Stecker herzustellen, künftig nur noch zwei oder drei zu produzieren hat. Schließlich lockt ein Markt mit 320 Millionen Euro-Konsumenten - satte 140 Millionen Konten mehr als der riesige nordamerikanische Binnenmarkt vorweisen kann. Es werden im Zuge der Euro-Deregulierung hinderliche und kostspielige nationale Verordnungen wegfallen. Euro-Pässe 
und Euro-Air, Euro-Car und Euro-Computer versprechen grenzenlose Bewegungsfreiheiten. Das Zuchtwunder Euro-Schwein wird endlich dem »brudermörderischen Kampf zwischen holländischen und französischen Schweinen« (E. Morin) ein Ende setzen und Orientierung in den Schweineberg bringen. Ganz Europa kann bald das zur Euro-Tomate veredelte holländische Nachtschattengewächs gleichen Namens genießen; und nur eine grünschnäbelige Minderheit wird den Etikettenschwindel bemerken.

Euphorien über Europa? Die ökonomischen Grenzen sollen fallen, aber was wird aus dem Machtmonopol der Nationalstaaten? Staaten ohne abgegrenztes Staatsgebiet gegenüber anderen Nationalstaaten, das ist auch für die europhilsten der EuropaPolitiker eine abwegige Vorstellung. Also werden schon jetzt vorsorglich neue Kontrollmechanismen installiert, durch die sich Staatsmacht in Szene zu setzen vermag und Innenminister (eine rïckständige Bezeichnung) sich profilieren können. Eine militärische Großmacht für Westeuropa muß her - und Euro-control soll nicht mehr an den Grenzen des Staatsgebietes enden. Mit den wirksamen Mitteln der elektronischen Fahndung kann sie genauso gut oder gar besser flächendeckend ausgeübt werden; so sieht es das »Schengener Abkommen « zwischen den Benelux-Ländern, Frankreich und der BRD vor. Mit dem Ziel einer $\gg$ Harmonisierung der Einreisebestimmungen « haben Innenminister und EG-Bürokratie - unter weitgehendem Ausschluß von Öffentlichkeit und nationalen Parlamenten - schon im Jahre 1985 mit dem Ausbau einer Art »Europäischem BKA « sowie eines »Europäischen FBI« begonnen. Auch Spanien, Italien und Österreich bemühen sich seither redlich um eine Aufnahme in diese Keimform einer »Europäischen Fahndungsunion «: Die Freiheit des grenzüberschreitenden Verkehrs also ein Vehikel zur Beförderung polizeilicher Kontrolle der europäischen Bürger. Parallel zu den Schengener Absprachen über grenzüberschreitende polizeiliche Ermittlungsgruppen werden auf den - ebenfalls ohne jede öffentliche Kontrolle ablaufenden - TREVI-Konferenzen polizeistaatliche Maßnahmen zur Terrorismus- und Drogenbekämpfung geplant. Sicher, auch die illegale EuroWirtschaft bereitet sich auf 1992 vor; die »unternehmerische Mafia « (P. Arlacci) aus Italien, deren Geschäftsvolumen allein für die Zeit von 1982-1984 auf 150 bis 200 Mrd. DM geschätzt wird, aber auch die Londoner und Amsterdamer Drogen-connections, die Waffenschieber aus deutschen Konzernzentralen und die Internationale der Giftmülltransporteure. Doch deren Geschäfte, so steht zu befürchten, werden so leicht nicht im Raster der Euro-Fahndung hängenbleiben; verfügen die kriminellen Netzwerke doch längst über Mittel und Wege, beispielsweise die schmutzigen narcolire in den Finanzmetropolen der legalen Ökonomie reinzuwaschen und sie dann in die internationalen Finanzkreisläufe einzufüttern.

Die politische Einigung des Rest-Kontinents (ohne die osteuropäische Brücke zu »Eurasien«) ist zweischneidig - die Herstellung des gemeinsamen Marktes etwa nicht? Der von der Kommission der Europäischen Gemeinschaft in Auftrag gegeben Cecchini-Bericht verbietet jeden Zweifel an euro-euphorischen Visionen. Er stellt wenigstens 1800000 neue Arbeitsplätze in Aussicht, die Belebung des Handels (durch Abschaffung von Zollformalitäten), mehr Investitionen und Konsum (durch 
billige Kredite für die Wirtschaft und die Verbraucher), Steuersenkungen und öffentlich finanzierbare Arbeitsplätze (durch mehr Konkurrenz bei den öffentlichen Aufträgen), größere Stückzahlen für die Unternehmen (durch den erweiterten Markt) und eine Verbesserung der Wettbewerbsfähigkeit europäischer Unternehmen auf dem (außereuropäischen) Weltmarkt. Doch sind hier Fragen zu stellen, beispielsweise die grundsätzliche: Was ist eigentlich ein gemeinsamer, einheitlicher Markt? Schon jetzt ist ja der innereuropäische Handel mit einem Drittel des gesamten Welthandels höchst bedeutsam. Die BRD etwa verkaufte 1988 mit 308,2 Mrd. DM mehr als die Hälfte ihrer Gesamtexporte (567,8 Mrd. DM) in Westeuropa. Der Außenhandelsüberschuß in der Größenordnung von $128 \mathrm{Mrd}$. DM ist mit 80,8 Mrd. DM zu fast drei Vierteln gegenüber europäischen Nachbarn erzielt worden. Was ist angesichts dieser bereits bestehenden Handelsverflechtungen ein gemeinsamer Markt, was seine besondere Qualität?

Nun, die Gemeinschaft der sechs umfaßte noch Länder mit einer ähnlichen ökonomischen Entwicklung, und das bedeutete in erste Linie intraindustriellen Austausch. Regionen in den einzelnen Ländern, der Mezzogiorno in Italien, das französische Zentralmassiv oder das bayrische Grenzland, blieben zwar zurück, doch war dies weniger ein europäisches als nationales Problem. Schließlich waren die Währungsräume national abgegrenzt und Entwicklungsunterschiede mußten durch Ressourcentransfer in den jeweiligen Ländern auszugleichen versucht werden. Bundesbankdirektor Leonhard Gieske bemerkt: »Ich erinnere mich an eine Diskussion im Währungsausschuß der EG Ende der fünfziger Jahre über Italien, in der unsere damaligen italienischen Kollegen mit Bezug auf die Entwicklungsprobleme des Mezzogiorno und des deshalb notwendigen Mitteltransfers vom Norden in den Süden... davon sprachen, daß die Standortnachteile des Südens leichter zu überwinden wären, wenn diese über einen Wechselkurs ausgeglichen werden könnten. In einem politischen Staatsgebilde wie Italien stand eine solche Währungsgrenze gewiß nicht zur Debatte. Aber das Argument beleuchtet doch die Probleme, mit denen eine Währungsunion konfrontiert wäre...«.

Der Handel unter zwölf Ländern mit unterschiedlichem ökonomischen Niveau und einheitlicher Währung (bzw. fixierten Kursen) hingegen könnte ganze nationale Industriezweige zum Erliegen bringen - vielleicht noch am wenigsten in der BRD, deren Außenhandel mit der EG von einer ungebrochenen Tendenz zum intra-industriellen Austausch geprägt ist. Der »Standort BRD « wird seine Attraktivität auch in einem vereinigten Europa nicht verlieren, auch wenn von Unternehmern der Teufel des Verlusts der Wettbewerbsfähigkeit an die Wand gemalt wird. Zu rechnen ist damit, daß eler gemeinsame Binnenmarkt zu einer weiteren Spezialisierung einzelner Länder auf bestimmte Industriesektoren führen wird. Eine kontinentale Umstrukturierung von Branchen und Regionen steht an.

Das »Europa der vielen Geschwindigkeiten« (P. Virilio) ist Realität, schon heute, vor der Zielmarke 1992: Spanien und Portugal haben mit einem rasanten Tempo den Einstieg in den Akkumulationstypus der »fordistischen Industrialisierung « vollzogen, in Ländern wie Frankreich und der Bundesrepublik oder Italien werden dagegen erste, 
langsame Schritte zum Ausstieg aus eben diesem Rationalisierungsmodell versucht. Durch den von der Binnenmarktintegration ausgehenden Modernisierungsschub holen die südlichen EG-Partner zugleich mit atemberaubender Geschwindigkeit die in den nördlichen und westlichen Nationen schon weit fortgeschrittene fordistische Naturzerstörung nach - derweil im Norden und Westen der EG sich gesellschaftliche Kräfte formieren, die eher auf einen ökologischen Umbau der nationalen Ökonomien setzen und dabei eines Tages das Tempo der Akkumulation von Kapital zu verlangsamen versuchen.

Sie begeben sich dabei in Widerspruch zu mächtigen Interessen. Denn dem europäischen Finanz.kapital kann die Tempodrosselung nicht gelegen sein; sein Prinzip, in der Zinseszinsformel ausgedrückt, ist die Beschleunigung. Integrieren wird der gemeinsame Markt gerade bei den Finanzdiensten, den Banken, dem Wertpapierhandel und bei den Versicherungen. Heute weisen die Preise dieser »Dienste « innerhalb der EG hohe Streuungen auf; morgen werden diese reduziert sein, wenn denn die Cassa di risparmio di Milano in München, die Stadtsparkasse von Köln in Marseille und Credit Lyonnais in Berlin ihre Dienste anbieten kann und erst recht, wenn zur »Krönung« der Vereinheitlichung des Marktes auch die Währungsdivergenzen verringert sein werden. So entsteht aber ein starker Druck in die Richtung einer Angleichung der Produktionskosten, d.h. der nationalen Regelungen von Produktion, Arbeitsorganisation, von Lohnverhältnissen und staatlicher Einflußnahmen auf die Produktivitäten. Kein Wunder, wenn der Daimler-Chef E. Reuter einen Lohnstopp für die BRD empfiehlt oder wenn inzwischen ein echt »europäisches Kulturgut « - der freie Sonntag nämlich - der Europa-Euphorie geopfert werden soll. Denn »Vereinheitlichung《 in diesem Sinne heißt zunächst nichts anderes als Anpassung der Kosten der Produktion an das europäisch niedrigste Niveau.

Die Produktivität der Arbeit und die Lohnkosten lassen sich im Index der Lohnstückkosten quantitativ und einfach ausdrücken, doch spiegelt dieser Index komplizierte soziale Verhältnisse wider: den Modernisierungsgrad der Wirtschaft, die Branchenstruktur, die Regelungen der Sozialleistungen durch den Staat, die Stärke der Gewerkschaften und die Form der Organisation der industriellen Beziehungen. Diese Verhältnisse sind auch in einem wirtschaftlich integrierten Europa durch die nationale Geschichte geprägt, und sie lassen sich nur höchst unzureichend mit dem juristischen Akt der Vereinheitlichung von Regelungen, d.h. zunächst: mit De-Regulierungen nationaler Regeln, zu einer einheitlichen Wirtschaft Europas zwingen. Modernisierungsvorsprünge, die einige Länder gegenüber anderen haben, lassen sich darum nicht umstandslos gegen die Lohnkostenvorteile der anderen verrechnen. Lohnkosten sind nur ein Argument unter vielen anderen für Standortentscheidungen von transnational operierenden Unternehmen. Andere Faktoren sind häufig von entscheidenderer Bedeutung, zum Beispiel die technologische und arbeitsorganisatorische Entwicklung der Unternehmen, die Qualifikation der Erwerbstätigen, finanzielle Gegebenheiten oder die bestehende Infrastruktur. Geschieht eine solche »Verrechnung « dennoch und wird versucht, der stagnierenden Arbeits- und Kapitalproduktivität durch »soziales Dumping « in den Hochlohnländern des Nordens und Westens 
Europas auf die Sprüne zu helfen, so nur um den Preis sozialer Desintegration. Dies mag aus der Sicht einzelner Unternehmen, die den Joker des innereuropäischen Standortwettbewerbs bei jeder Gelegenheit ins Spiel bringen, eine zu vernachlässigende Größe sein; in einer makroökonomischen und längerfristigen Perspektive wäre soziale Desintegration glerichwohl ruinös für das europäische Vereinheitlichungsprojekt. Auch hier wieder: Der Widerspruch zwischen privatwirtschaftlicher und gesamtwirtschaftlicher, d.h. heute europäischer Rationalität.

Den marktwirtschaftlichen Befürwortern des Binnenmarktes, insbesondere jenen aus der reichen BRD, ist die Vision eines »sozialen Europas « eine greuliche Vorstellung. So befürchtet beispielsweise $H$. Giersch vom Kieler Institut für Weltwirtschaft, »populäre Wohltaten« könnten die »Nassauer« aus den ärmeren Mitgliedsstaaten anlocken; dagegen hilft dann nur »eine weise Beschränkung « in Sachen Umverteilungspolitik. Eine Nivellierung aller nationalen und branchenspezifischen Differenzen in den Arbeitszeiten, Löhnen, bei den Regelungen der industriellen Beziehungen oder der sozialstaatlichen Transfereinkommen wäre das letzte, was diese Streiter für »realistische Preis-Leistungs-Verhältnisse sich vom Binnenmarkt erhoffen. Wollen sie sich doch die Möglichkeiten nicht nehmen lassen, in jenem Lande »down-grading «Strategien in bezug auf die Qualifikationen der Arbeitskräfte zu verfolgen, in einem anderen aber eher »up-grading «-Strategien; hier starke Gewerkschaften kleinzukriegen, dort sie politisch zu isolieren oder als Partner im internationalen Verdrängungswettbewerb einzuspannen; in den südlichen Ländern die Herstellung arbeitsintensiver Produkte mit niedrigen Löhnen zu entgelten, im Norden und Westen die störanfällige Herstellung technologieintensiver Produkte aber durch hohe Löhne sicherzustellen. Mit der 10. EG-Richtlinie zur »Erleichterung grenzüberschreitender Fusionen von Aktiengesellschaften in der Gemeinschaft « verbinden insbesondere die westdeutschen Gewerkschaften eine Horrorvision: sie könnte zum Wegfall hier geltender tariflicher Schutzbestimmungen und institutionalisierter Mitbestimmungsrechte führen, die Anwendbarkeit deutschen Arbeitsrechts, die Zuständigkeit deutscher Arbeitsgerichte infragestellen, die Mitbestimmung auf Konzern- und Unternehmensebene gefährden. Dagegen steht aber die keineswegs unwahrscheinliche Einschätzung, daß die BRD eher zum ökonomischen Nutznießer des EG-Binnenmarktes werden, das deutsche Modell gewerkschaftlicher Interessenvertretung und Mitbestimmung über die heutigen Landesgrenzen hinweg expandieren könnte - eine aus der Sicht anderer europäischer Gewerkschaften keineswegs nur erfreuliche Perspektive.

Von einer Gleichgerichtetheit der Mitgliederinteressen in einem wirtschaftlich stärker als bisher schon integrierten Europa kann aber auch aus anderen Gründen keine Rede sein: Das »Starkwährungsland « BRD, das im Währungsverbund der Gemeinschaft das Sagen hat, setzt auf Geldwertstabilität und wenig spricht dafür, daß die konservative Regierungsmehrheit in Bonn und die Bundesbank bereit sind, um der europäischen Einheit willen von ihrer restriktiven Geldpolitik Abschied zu nehmen. Andere Partnerländer wie Frankreich und Italien, die mit höheren Inflationsraten zu kämpfen haben und eher auf Wachstumsanreize resp. möglichst hohe Beschäftigungsgewinne orieniert sind, werden aber-um ihre Währungen im Verbund stabil zu 
halten - zu höheren Zinssätzen gezwungen; ihnen muß eine europäische Zentralbank als ein notwendiger Schritt zur Verteidigung ihrer Währungen gegen die $\gg D M \ll$ erscheinen. Von einem weiteren Ausbau des europäischen Währungssystems aber wollen Bonn und die Bundesbank so lange nichts wissen, wie nicht in Frankreich, Italien und Belgien alle Beschränkungen des Kapitalverkehrs aufgehoben sind und »Stabilitätspolitik « betrieben wird.

Mit einem Satz: Die »krasse Utopie des Marktes« (K. Polanyi) wird wohl vorerst in Europa nicht realisiert - dieses Projekt in Aktion wäre auch katastrophal. Ist doch mittlerweise absehbar, wie viele Verlierer der freie europäische Markt »produzieren wird: wirtschaftsschwache Branchen und Regionen in Europa wie in der Dritten Welt müssen ihn fürchten, aber Bedenken äußern auch die Verfechter einer sozialen Demokratie - von den Ökologen ganz zu schweigen. Regulierung der Arbeit, des Umgangs mit der Natur, mit der inner- und außereuropäischen »Peripherie« ist verlangt, nicht die weitere Entregelung des Spiels der Marktkräfte. Mit der Erweiterung des Binnenmarktes wird sich das Wohlstandsgefälle in der Gemeinschaft keinesfalls nivellieren. Ganz im Gegenteil: In Portugal beispielsweise ist der Boom der letzten Jahre ins Stocken geraten, flacht sich die Investitionskurve ab, klettert das Handelsbilanzdefizit in schwindelerregende Höhen, liegen die Durchschnittslöhne - mit Ausnahme der Großstädte Lissabon und Porto sowie der Touristikzentren - weiterhin unter der 1.000 DM-Grenze, ist von einem Abbau der Massenarbeitslosigkeit nichts zu spüren. Kaum anders sieht es im derzeitigen Musterland europäischer Modernisierungspolitik, in Spanien, aus. Auch hier ist ein Viertel der Erwerbstätigen arbeitslos, bei den Jugendlichen sogar knapp die Hälfte; leben dreißig Prozent der Bevölkerung unterhalb der Armutsschwelle. Auch hier hat sich in den vergangenen Jahren eines beispiellosen wirtschaftlichen Aufschwungs keine im europäischen Maßstab konkurrenzfähige Wirtschaft herausgebildet. Auch die griechische Bevölkerung oder die von der grünen Insel Irland weiß, daß vor den Euphorien über den EG-Binnenmarkt zunächst die Milchseen ausgetrocknet, die Schweineberge abgetragen und mehr Hektoliter ihres Weins getrunken werden müssen. Um die für eine »gesunde« Wirtschaft so wichtige Infrastruktureinrichtung des Verkehrs, der Kommunikation, der Energieversorgung und des Bildungswesens auch nur annähernd auf den EGDurchschnitt zu bringen, müßten jährlich bedeutend mehr Mittel zur Verfügung gestellt werden als die Gemeinschaft derzeit für ihre Regional- und Sozialfonds ausgibt. Einer der Gründe, weshalb wirtschaftlich schwache Regionen und Branchen von der Binnenmarktintegration so wenig erwarten können, ist der, daß viele Investitionen, die in Ländern wie Spanien, Portugal, Griechenland, Irland getätigt werden, die regionalen Ungleichheiten in diesen Ländern eher noch verschärfen und gerade nicht dazu verhelfen, eine »gesunde «nationale Wirtschaftsstruktur zu entwickeln. Bleiben aber die Unterschiede oder verschärfen sie sich gar, dann ist die Währungsunion, die monetäre Krönung des gemeinsamen Marktes, eine »krasse Utopie«, Katalysator sozialer Konflikte.

Ohne eine wirksame europäische Fusionskontrolle, die dem ruinösen Spiel der Euromergers Einhalt gebietet, werden nicht nur die weniger entwickelten Länder zu 
Opfern eines $»$ Europas der Konzerne«. Denn die Übernahmeschlachten um die belgische Holding Société Générale, der Einstieg des finnischen Telekommunikationsriesen Nokia in den Unterhaltungselektronikbereich der deutschen SEL, die von Berlusconi und Murdoch betriebene Konzentration in der europäischen Medienlandschaft, die Cognac-, Zement-, Zucker- und Versicherungsschlachten, nicht zu vergessen die Konzentrationen im europäischen Rüstungsgeschäft, mit denen die großen Konzerne schon heute ihre claims in der »Europa $A G \ll$ abstecken, werden entgegen allen anderslautenden Euphorien mitnichten $»$ nur « die Kapitalproduktivität verbessern und den Prozeß der Produkt- und Prozeßinnovation beschleunigen. Ob nun der englische Elektronikkonzern GEC oder die bundesdeutsche Siemens AG den Plessey-Konzern in Großbritannien übernehmen oder ob Plessey zusammen mit einem Konsortium anderer Unternehmen die GEC übernimmt - im Endeffekt läuft das auf dasselbe hinaus: Die Kontrolle von Schlüsselbereichen der englischen elektrotechnischen und Elektronikindustrie wird sich - entweder in finanzieller oder in technologischer Hinsicht - von dem nationalen Unternehmen auf ein ausländisches (die bundesdeutsche Siemens AG, den us-amerikanischen Telekommunikationsriesen GE und/oder den französischen Elektrokonzern Thomson CSF) verlagern. Die Konsequenzen dieses Restrukturierungsprozesses der europäischen Elektro- und Elektronikindustrie aber werden wohl auf jeden Fall in negative Beschäftigungseffekte einmünden - denn, so formulierte es ein Sprecher von Swedish Swiss ABB, ein GECKonkurrent: »Die harten Realitäten hinter den hübschen Worten höhere Produktivität und mehr Wettbewerb sind eine Menge weniger Beschäftigte «!

Verlieren könnte durch ein unreguliertes Spiel der Marktkräfte aber nicht zuletzt auch eines der wichtigsten »europäischen Kulturgüter«, auf das gerade die sozialdemokratischen Verfechter der europäischen Einigung ihre Modernisierungsbereitschaft gründen: die parlamentarische Demokratie. Solange wie der Rat, der aus weisungsgebundenen Regierungsmitgliedern aller Mitgliedsstaaten sich zusammensetzt, das einzige gesetzgebende Organ bleibt, Richtlinien und Verordnungsvorschläge allein die EG-Kommission macht, auf deren Vorschlag der Rat handelt und das EuropaParlament samt Wirtschafts- und Sozialausschuß gerade einmal Stellungnahmen zu den Gesetzesinitiativen abgeben kann, so lange werden die heute noch existierenden Grenzen Europas niedergerissen, ohne daß den 320 Mio. EG-Bürgern transnationaler, europäischer Gestaltungsspielraum zuwachsen würde. Friedensbewußte EGBürger in der Bundesrepublik und in den Niederlanden werden auf EG-Ebene lange nach Bündnispartnern Ausschau halten müssen, die mit ihnen gegen eine westeuropäische Militärstrategie optieren, deren einziges Ziel die Teilhabe an einer Gestaltung der »Weltordnung « ist. Für eine fortschrittliche Umweltpolitik gar scheint Brüssel der denkbar schlechteste Ort: Was immer in nationaler Regie zum Schutze von Wasser und Luft auf parlamentarischem Wege auch erzwungen werden könnte-im »gemeinsamen Haus Europa « wird sich so manch eine Umweltsünde eurokratisch legitimieren und in den Keller kehren lassen. Wird Europa also scheitern - oder doch noch ein neues System von menschen- und umweltverträglichen Regelungen schaffen, inclusive eines gewaltigen internen Finanzausgleichs, zum Ausgleich für den Schutz durch 
national unterschiedliche Regelungen? Müssen wir also im Zeitalter der Dezentralisierung von allem und jedem auf eine große und starke Bürokratie unsere letzten Hoffnungen setzen?

Ein modernisiertes und rationalisiertes Europa, das zumindest ist leider keine Spekulation mehr, hat mit der Entstehung atavistischer Gegenbewegungen zu rechnen. Schon heute sitzen zehn Abgeordnete der französischen Front National, sechs vom italienischen MSI und ein Vertreter der griechischen Rechtsradikalen im Europaparlament. Nach den Europawahlen im Juni 1989 wird damit gerechnet, daß diese Rechtsparteien durch Vertreter aus Dänemark, Spanien und der Bundesrepublik Verstärkung erhalten - und es steht zu befürchten, daß es zumindest auf der programmatischen Ebene zu einem Bündnis zwischen Rechtsradikalen und konservativem Bürgerblock im Europaparlament kommen könnte. Denn die Euro-Rechte, deren Chauvinismus aus Fremdenhaß, auch Anti-Semitismus sich nährt, »nimmt die regierende Rechte beim Wort, (...) spricht aus, was andere denken« (W. Hoffmann). Sie ficht mit der sogenannten Asylantenfrage einen Windmühlenkampf: Es ist ein Kampf der von sozialem Abstieg bedrohten Schichten gegen die internationale Arbeitsteilung, die die westeuropäischen Staaten unwiderruflich zu Einwanderungsgesellschaften transformiert hat - und er wird ausgetragen auf dem Rücken inner- und außereuropäischer Flüchtlinge. Sie sind dabei zum einen Stellvertreter für die Dritte Welt, deren ökonomische, soziale und politische Katastrophe von den Industrieländern mitverschuldet ist, für die diese auch die Mitverantwortung zu tragen haben. Als »weniger europäische Europäer « sind die Einwanderer zugleich die sichtbarste soziale Gruppierung des letzten Drittels der »informellen« Gesellschaft, die die Zwei-Drittel förmlicher Gesellschaft durch Lebensweise und Kultur in jeder Hinsicht infragestellt.

Wenn es nicht gelingt, in der Asylpolitik der europäischen Staaten jene Grundsätze zu verankern, welche in der Genfer Konvention von 1951 und in der europäischen Menschenrechtskonvention verankert sind, wird das »Europa 1992《 in der Tat einer »Festung « gleichen: Freiheit der Waren- und Kapitalzirkulation und Wälle gegen die Migration von Menschen. Dies ist jedoch keine Festung, wie sie derzeit von den USA und Japan befürchtet wird - sichert sich das internationale Kapital durch Direktinvestitionen doch längst schon sein Plätzchen auf dem Binnenmarkt. Die »Festung Europa « wird dann einzig und allein unerwünschte Einwanderer fernhalten, nicht nur die aus der Dritten Welt sondern ebenso jene aus der Zweiten Welt des »realen Sozialismus«, mit der - »Perestroika« sei dank - zwar joint ventures erwünscht sind, deren Migranten aber mit der Waffe einer biologistisch eingefärbten nationalen Gemeinschaftsrhetorik von den Arbeitsplätzen, den Wohnungen und Schulen des reichen Europas ferngehalten werden sollen. Das aber wäre 200 Jahre nach der Erklärung der Menschenrechte und dem Bicentenaire der Französischen Revolution und fast 500 Jahre nach der Eroberung der »neuen Welt« durch die Spanier ein beschämendes Zeugnis jener »europäischen Kultur«, über die aus der Perspektive aller NichtEuropäer dann wohl zu recht gesagt werden müßte: »Europa hat uns nichts mehr zu sagen. Europa hat nichts mehr zu sagen« (E. Galeano). 